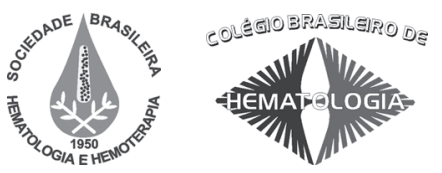

\title{
Protocolo clínico e diretrizes terapêuticas para uso de hidroxiureia na doença falciforme
}

\author{
Clinical protocol and therapeutic guidelines for the use of hydroxyurea in sickle cell disease
}

\author{
Rodolfo D. Cançado ${ }^{1}$ \\ Clarisse Lobo ${ }^{2}$ \\ Ivan L. Angulo ${ }^{3}$ \\ Paulo I. C. Araújo \\ Joice A. Jesus ${ }^{5}$
}

\begin{abstract}
Hidroxiureia (HU) constitui o avanço mais importante no tratamento de pacientes com doença falciforme (DF). Fortes evidências confirmam a eficácia da HU em pacientes adultos diminuindo os episódios de dor intensa, hospitalização, número de transfusões e síndrome torácica aguda. Embora a evidência da eficácia do tratamento com HU em crianças não seja tão forte, os recentes resultados são encorajadores. Os dados atuais em relação aos riscos a curto e longo prazos da terapia com $\mathrm{HU}$ em adultos são aceitáveis comparado aos riscos dos pacientes não tratados com HU. Neste artigo, apresentamos revisão detalhada sobre os principais aspectos quanto à eficácia, efetividade, toxicidade e barreiras ao uso de HU em pacientes com DF e propomos um protocolo clínico e diretrizes terapêuticas para o uso de HU em pacientes com DF. Rev. Bras. Hematol. Hemoter.
\end{abstract}

Palavras-chave: Doença falciforme; hidroxiureia; protocolo clínico; diretrizes terapêticas.

\section{Introdução}

Situação atual da doença falciforme

Anemia falciforme (AF) é a doença hereditária monogênica mais comum do Brasil, ocorrendo predominantemente entre afrodescendentes. Distribui-se heterogeneamente, sendo mais frequente nos estados Norte e Nordeste. Estimase que cerca de $4 \%$ da população brasileira e $6 \%$ a $10 \%$ dos afrodescendentes são portadores do traço falciforme (Hb AS) e que, anualmente, nascem aproximadamente 3 mil crianças portadoras de doença falciforme (DF), número este que corresponde ao nascimento de uma criança doente para cada mil recém-nascidos vivos. Atualmente, estima-se que tenhamos 20 a 30 mil brasileiros portadores da DF. ${ }^{1,2}$
O evento fisiopatológico básico da AF é a mutação de ponto (GAG por GTC) no gene da globina beta da hemoglobina $(\mathrm{Hb})$, originando a formação da $\mathrm{Hb} \mathrm{S}$, que, em situação de desoxigenação, sofre polimerização de suas moléculas, com falcização das hemácias, ocasionando encurtamento da vida média dos glóbulos vermelhos (anemia hemolítica crônica), eventos repetidos de vaso-oclusão, episódios de dor e lesão crônica e progressiva de órgãos, resultando em piora da qualidade de vida e aumento da taxa de mortalidade. ${ }^{3}$

Até o momento presente, a hidroxiureia (HU) foi o único medicamento que, efetivamente, teve impacto na melhora da qualidade de vida dos pacientes com $\mathrm{DF}$, reduzindo o número de crises vaso-oclusivas, número de hospitalização, tempo de internação, ocorrência de STA e, possivelmente, de eventos

\footnotetext{
${ }^{I}$ Médico Hematologista, Professor Ajunto da Disciplina de Hematologia e Oncologia da F.C.M. da Santa Casa de São Paulo.

${ }^{2}$ Médica Hematologista, Diretora Geral do Hemorio - Rio de Janeiro-RJ

${ }^{3}$ Assessor médico da Fundação Hemocentro de Ribeirão Preto, Médico do Centro Regional de Hemoterapia do Hospital das Clínicas da Faculdade de Medicina de Ribeirão Preto da USP.

${ }^{4}$ Médico Hematologista do Instituto de Puericultura e Pediatria Martagão Gesteira (IPPMG) da UFRJ - Rio de Janeiro-RJ

${ }^{5}$ Médica Pediatra sanitarista, coordenadora da Política Nacional de Atenção Integral às Pessoas com Doença Falciforme e outras Hemoglobinopatias do Ministério da Saúde do Brasil.
}

Disciplina de Hematologia e Oncologia da F. C. M. da Santa Casa de São Paulo - São Paulo-SP.

Correspondência: Rodolfo Delfini Cançado

Rua Marques de Itu, $579-3^{\circ}$ andar

01223-001 - São Paulo-SP - Brasil

E-mail:rdcan@uol.com.br

Doi: 
neurológicos agudos, além de demonstrar, de maneira contundente, redução da taxa de mortalidade quando comparada à mesma taxa no grupo de pacientes sem $\mathrm{HU}^{4,5}$

\section{Hidroxiureia (HU)}

A HU foi sintetizada, pela primeira vez, na Alemanha, por Dressler e Stein, em 1869. ${ }^{6}$ Somente um século depois, mais especificamente em 1967, este medicamento foi aprovado pelo FDA norte-americano para tratamento de doenças neoplásicas e, nos anos subsequentes, para o tratamento de pacientes com leucemia mieloide crônica, psoríase e policitemia vera. ${ }^{?}$

A observação de que valores aumentados de $\mathrm{Hb}$ fetal ( $\mathrm{Hb} \mathrm{F})$ previnem várias complicações da DF conduziu os pesquisadores à busca por fármacos que estimulassem a síntese de cadeias globínicas gama e aumentassem a síntese intraeritrocitária de $\mathrm{Hb} \mathrm{F}^{7}$

A partir de fevereiro de 1998, a HU passou a fazer parte do arsenal terapêutico para pacientes com DF e se tornou, nos anos subsequentes, o primeiro medicamento que comprovadamente previne complicações clínicas, melhora a qualidade de vida e aumenta a sobrevida de pacientes com DF. ${ }^{8-10}$

Além da vantagem de administração oral, HU é um medicamento seguro, de fácil controle; apresenta poucos efeitos adversos e efeito mielossupressor facilmente detectável e reversível após a suspensão do uso da mesma. Diante da eficácia e efetividade da HU na DF, vários autores têm se manifestado a favor do uso deste medicamento, salientando que os riscos relacionados às complicações secundárias à DF são muito mais elevados e graves que os riscos relacionados aos efeitos adversos da HU. ${ }^{10}$

O mecanismo de ação da HU não é totalmente conhecido. Ela promove bloqueio da síntese do ácido desoxirribonucleico (DNA) pela inibição da ribonucleotídeo redutase, mantendo as células em fase $\mathrm{S}$ do ciclo celular. ${ }^{11}$

A HU tem vários efeitos diretos no mecanismo fisiopatológico da DF atuando não só no aumento da síntese da $\mathrm{Hb} \mathrm{F}$, o que reduz a polimerização intraeritrocitária da $\mathrm{Hb} \mathrm{S}$ em condições de desoxigenação, como também promove diminuição do número dos neutrófilos, hidratação eritrocitária, redução da expressão de moléculas de adesão dos eritrócitos, aumento da síntese e biodisponibilidade de óxido nítrico pela ativação da guanilil ciclase e consequente aumento da GMP cíclico intraeritrocitária e endotelial. Além do aumento da concentração de $\mathrm{Hb} F$, estes mecanismos conferem benefícios como: supressão da eritropoese endógena, redução da hemólise, diminuição da aderência dos eritrócitos, leucócitos e plaquetas ao endotélio vascular (HU age sobre a membrana dos eritrócitos e plaquetas, reduzindo a exposição da fosfatidilserina, principal determinante da adesão eritrocitária alterada na AF, melhora da reologia com diminuição da viscosidade sanguínea e vasodilatação, contribuindo para a diminuição dos fenômenos inflamatórios e vaso-oclusivos. ${ }^{12-21}$
Embora estes dados correspondam à eficácia da HU em adultos, os poucos estudos publicados em crianças demonstram eficácia, tolerância e segurança semelhantes aos encontrados no adulto. ${ }^{21-26}$ Dois importantes estudos, Baby Hug ${ }^{27}$ envolvendo 200 crianças com DF, randomizadas em HU versus observação, com avaliação da função esplênica e renal; e o Switch, ${ }^{28}$ envolvendo 130 crianças pós-acidente vascular cerebral, em transfusão regular, randomizadas em HU e flebotomia versus manutenção das transfusões e deferasirox, com avaliação das funções neurológica e cognitiva, trarão contribuição importante do uso de $\mathrm{HU}$ em crianças.

A HU administrada por via oral é rapidamente absorvida atingindo nível plasmático máximo entre 20-30 minutos (respondedores rápidos) e 60 minutos (respondedores lentos) após sua administração e meia vida plasmática de três a quatro horas, é metabolizada no fígado e excretada por via renal $(80 \%){ }^{29-31}$ Recomenda-se dose inicial de $15 \mathrm{mg} / \mathrm{kg} / \mathrm{dia}$, uma única vez ao dia, e monitoramento da contagem do número de leucócitos e plaquetas (hemograma completo) a cada duas semanas. Esta dose inicial pode ser aumentada de $5 \mathrm{mg} / \mathrm{kg} /$ dia a cada oito a 12 semanas, sendo que o objetivo é alcançar a dose máxima tolerada (DMT), isto é, a maior dose capaz de promover melhora o mais proeminente possível do curso clínico e laboratorial da doença, sem a ocorrência de toxicidade hematológica, hepática (definida por aumento de duas vezes o valor referencial máximo das transaminases), renal (elevação da ureia e creatinina) ou gastrointestinal. A DMT não deve ser superior a $35 \mathrm{mg} / \mathrm{kg} /$ dia. ${ }^{29-31}$

Em adultos portadores de AF, a HU é potencialmente mielossupressora, sendo este efeito dose-dependente. Estudos experimentais em ratos e cães demonstraram a ocorrência de hipoplasia medular de intensidade leve a moderada com o uso de 34 a $60 \mathrm{mg} / \mathrm{kg} /$ dia e hipoplasia grave e, por vezes, fatal, com doses superiores a $140 \mathrm{mg} / \mathrm{kg} / \mathrm{dia}$. A recuperação medular, nos casos de hipoplasia leve a moderada, é rápida após a suspensão da droga. Também observou-se que a trombocitopenia ocorre mais raramente e é sempre precedida de anemia e leucopenia.

Além da redução do número de crises vaso-oclusivas, o uso crônico de HU teve impacto positivo na qualidade de sobrevida dos pacientes com $\mathrm{AF}$, com redução do número de hospitalização, tempo de internação, menor ocorrência de síndrome torácica aguda e menor necessidade de transfusão de hemácias. Estudos mais recentes têm indicado que o uso da HU tem impacto na redução da mortalidade de até $40 \%$ desses pacientes. ${ }^{29-31}$

No Brasil, a portaria de n 872 do Ministério da Saúde, de 6 de novembro de 2002, aprovou o uso de HU para pacientes com DF. Nesta portaria, a dispensa desse medicamento passou a ser de incumbência das Secretarias de Saúde dos Estados da União e Distrito Federal. . $^{32}$

Apesar dos vários benefícios anteriormente enumerados com o uso continuado da HU, este medicamento ainda é 
subutilizado em crianças, mas sobretudo nos adolescentes e adultos com DF. Esta constatação foi feita em pacientes norteamericanos com DF e, com grande certeza, também reflete a nossa realidade brasileira. Nos EUA, apenas $10 \%$ a $20 \%$ dos pacientes com DF são acompanhados em centros de excelência e, destes, menos da metade recebe HU. Estima-se que, fora dos centros de referência, o uso de HU seja ainda menor. ${ }^{29,30}$

$\mathrm{O}$ item segurança do uso de $\mathrm{HU}$ a longo prazo permanece como questão importante, sobretudo quanto à genotoxicidade e lesão celular, e impacto na função de diferentes órgãos (baço, rins, cérebro, pulmões). Desta forma, a realização de novos estudos clínicos são fundamentais e permitirão maior aceitação e uso da HU pelos pacientes e comunidade médica. ${ }^{29-31}$

\section{Protocolo clínico e diretrizes terapêuticas para uso de hidroxiureia na doença falciforme - versão 2009}

Critérios de inclusão

Poderão ser incluídos nesse Protocolo de Tratamento pacientes que preencham todos os critérios abaixo:

- Diagnóstico laboratorial de doença falciforme (HbSS, $\mathrm{S} /$ Talassemia, HbSC, Hb SD)

- Idade superior a 3 anos

- Condições de comparecer às consultas e realizar exames laboratoriais periódicos

- Teste de gravidez (ß-HCG sérico) negativo para mulheres sexualmente ativas, lembrando que a anticoncepção é obrigatória enquanto a paciente estiver fazendo uso de HU.

E pelo menos um dos seguintes critérios nos últimos 12 meses:

Critérios clínicos

- Três ou mais episódios de crises de dor com necessidade de atendimento médico hospitalar.

- Síndrome torácica aguda (definida como dor torácica aguda com infiltrado pulmonar "novo", febre (temperatura $>38,5^{\circ} \mathrm{C}$ ), taquipneia, tosse, sibilos pulmonares): dois ou mais eventos (leve a moderado)/ano ou um evento grave.

- Hipoxemia crônica: saturação de oxigênio persistentemente menor que $94 \%$.

- Déficit pôndero-estatural: peso e crescimento observados menores que $5 \%$ a $10 \%$ do esperado para a idade.

- Outras situações em que haja comprovação de lesão crônica de órgão (priapismo, necrose óssea, retinopatia proliferativa).

Critérios laboratoriais $7 \mathrm{~g} / \mathrm{dL}$.

- Concentração de $\mathrm{Hb}$ persistentemente menor que

- Concentração de $\mathrm{Hb}$ fetal $<8 \%$ após 2 anos de idade
- Contagem de leucócitos $20 \times 10^{9} / \mathrm{L}$ na ausência de infecção.

- Duas medidas consecutivas do fluxo das artérias cerebrais média ou anterior por Doppler tanscraniano com velocidade entre 170 e $199 \mathrm{~cm} / \mathrm{s}$ (inclui pacientes com história prévia de isquemia cerebral transitória e/ou acidente vascular cerebral que recusam tratamento transfusional regular ou que apresentam aloimunização com presença de múltiplos aloanticorpos).

- Desidrogenase láctica maior que duas vezes e três vezes o valor superior de normalidade para crianças e adolescentes e adultos, respectivamente.

Situação especial

Crianças com menos de 3 anos de idade com pelo menos um dos seguintes fatores:

- dactilite (antes do primeiro ano de vida).

- concentração de $\mathrm{Hb}$ persistentemente menor que $7 \mathrm{~g} / \mathrm{dL}$.

- contagem de leucócitos maior que $20 \times 10^{9} / \mathrm{L}$

Critérios de exclusão

Não deverão ser incluídos neste Protocolo de Tratamento pacientes com:

- Hipersensibilidade à HU.

- Presença de pelo menos um dos seguintes ítens relacionados à disfunção da medula óssea: neutrófilos $<2.5 \times 10^{9} / \mathrm{L}$, plaquetas $<95 \times 10^{9} / \mathrm{L}$, concentração de $\mathrm{Hb}$ $<4,5 \mathrm{~g} / \mathrm{dL}$ ou contagem de reticulócitos $<95 \times 10^{9} / \mathrm{L}$.

- Gestação (não há estudos adequados em humanos e há evidência de teratogênese em animais).

- Infecção pelo HIV.

\section{Tratamento}

Esquema de administração

A HU está disponível em cápsulas de gel sólido contendo $500 \mathrm{mg}$ do princípio ativo.

Recomenda-se dose inicial de $15 \mathrm{mg} / \mathrm{kg} / \mathrm{dia}$, uma única vez ao dia, e monitoramento da contagem do número de leucócitos e plaquetas (hemograma completo) a cada duas semanas. Deve-se considerar o peso real ou ideal, o que for menor. Para crianças, recomenda-se dissolver a cápsula de $500 \mathrm{mg}$ de HU em água destilada, obtendo a concentração de $50 \mathrm{mg} / \mathrm{mL}$, o que facilita a administração da dose correta por kilograma de peso. ${ }^{33}$

Desde que as contagens celulares estejam dentro dos valores considerados aceitáveis, ou seja, leucócitos $\geq 2.5 \mathrm{x}$ $10^{9} / \mathrm{L}$, plaquetas $\geq 95 \times 10^{9} / \mathrm{L}$, concentração de $\mathrm{Hb}>5,3 \mathrm{~g} / \mathrm{dL}$, ou reticulócitos $\geq 95 \times 10^{9} / \mathrm{L}$, se a concentração de $\mathrm{Hb}$ for menor que $9 \mathrm{~g} / \mathrm{dL}$; esta dose inicial pode ser aumentada de $5 \mathrm{mg} / \mathrm{kg} /$ dia a cada 8 a 12 semanas. O objetivo é alcançar a dose máxima tolerada (DMT), isto é, a maior dose capaz de promover melhora mais proeminente possível do curso clínico 
e laboratorial da doença sem a ocorrência de toxicidade hematológica, hepática (definida por aumento de duas vezes o valor referencial máximo das transaminases), renal (elevação da ureia e creatinina) ou gastrointestinal. A DMT não deve ser superior a $35 \mathrm{mg} / \mathrm{kg} / \mathrm{dia} .{ }^{29-31}$

Uma vez detectada toxicidade hematológica, ou seja, leucócitos $<2.0 \times 10^{9} / \mathrm{L}$, plaquetas $<80 \times 10^{9} / \mathrm{L}$, concentração de $\mathrm{Hb}$ menor que $4,5 \mathrm{~g} / \mathrm{dL}$, ou reticulócitos $<80 \times 10^{9} / \mathrm{L}$ com Hb menor que $9 \mathrm{~g} / \mathrm{dL}$, isto é, contagem de reticulócitos corrigida para o valor de $\mathrm{Hb}$; a $\mathrm{HU}$ deve ser descontinuada até a recuperação hematológica e, a seguir, reiniciada com dose $2,5 \mathrm{mg} / \mathrm{kg}$ menor que a dose que o paciente estava utilizando quando apresentou a intercorrência, seguindo os mesmos critérios de controle até a dose máxima tolerada para cada específico paciente, que pode ser de 20,25 ou $35 \mathrm{mg} / \mathrm{kg} /$ dia.

\section{Duração do tratamento}

O tratamento deve ser de, pelo menos, dois anos e mantido por tempo indeterminado de acordo com a resposta laboratorial e evolução clínica do paciente, exceto no período gestacional e puerperal.

É importante lembrar que cerca de $25 \%$ dos pacientes não apresentam melhora com $\mathrm{HU}$ e, portanto, nestes casos esse tratamento deve ser descontinuado.

\section{Beneficios esperados}

Diminuição dos episódios de dor, que podem até mesmo desaparecer; aumento da produção de $\mathrm{Hb}$ fetal; aumento, mesmo que discreto, da concentração total da $\mathrm{Hb}$; diminuição dos episódios de síndrome torácica aguda, do número de hospitalizações, do número de transfusões sanguíneas; regressão ou estabilização de danos em órgãos ou tecidos (baço, rins, cérebro, coração, pulmões), melhora do bem-estar e da qualidade de vida e maior sobrevida.

Aumento da $\mathrm{Hb}$ fetal e macrocitose são parâmetros laboratoriais úteis na análise de aderência do paciente ao tratamento.

\section{Monitorização laboratorial}

Realizar antes de iniciar o tratamento:

- Hemograma com contagem de plaquetas.

- Contagem de reticulócitos.

- Eletroforese de $\mathrm{Hb}$ com dosagem de $\mathrm{Hb}$ fetal.

- Sorologias: hepatite B e C e HIV.

- Determinação do tempo de protrombina (TP) e dosagem de transaminases (AST, ALT), fosfatase alcalina, gama-GT.

- Determinação do clearance de creatinina.

- Dosagem de ureia, creatinina, sódio, potássio e ácido úrico.

- Dosagem de bilirrubina.

- Dosagem de desidrogenase láctica.

- Dosagem de ferritina.
- Teste de gravidez em mulheres (B-HCG sérico). Devem ser reiterados os riscos em caso de gravidez e as orientações para o uso de métodos contraceptivos durante todo o tratamento.

Hemograma completo e contagem de plaquetas a cada duas semanas até obtenção da dose máxima tolerada. Com relação aos demais exames, estes deverão ser realizados mensalmente nos quatro primeiros meses, a cada três meses nos doze meses subsequentes e a cada três a seis meses nos anos subsequentes, ou de acordo com a necessidade de cada paciente.

Cuidados e precauções, situações especiais

Devido aos possíveis efeitos adversos da droga, a relação entre risco e o benefício deve ser cuidadosamente avaliada nos seguintes casos:

- Amamentação: sabe-se que o fármaco é excretado pelo leite. Não há estudos suficientes para determinar seus efeitos sobre o lactente. Seu uso deve ser evitado ou descontinuado durante a amamentação.

- Uricosúria: o uso de HU pode aumentar os níveis séricos de ácido úrico. Em pacientes com níveis basais acima do limite normal, estes valores devem ser monitorados mensalmente.

- Ácido fólico: o uso de HU produz macrocitose, dificultando o reconhecimento da deficiência de ácido fólico. Desta forma, é recomendado o emprego profilático concomitante de $5 \mathrm{mg} /$ dia de ácido fólico, três vezes por semana.

- Úlcera isquêmica é um possível efeito adverso do uso crônico de HU. Pacientes com história prévia ou atual de úlcera isquêmica, não há contraindicação formal do uso de $\mathrm{HU}$; entretanto, no aparecimento de úlcera isquêmica em pacientes sem história anterior desta complicação, a suspensão da HU deve ser considerada. ${ }^{34}$

- Interações medicamentosas: não há estudos adequados sobre interação entre $\mathrm{HU}$ e outros medicamentos. Portanto, o uso concomitante de outros fármacos, principalmente os que também possam produzir depressão da medula óssea, deve ser cuidadosamente monitorizado.

- Em pacientes com sorologia positiva para HIV, o uso de HU aumenta o risco de neuropatia periférica, pancreatite e insuficiência hepática, principalmente quando associados a antirretrovirais, como didanosina e estavudina. Portanto, nestes casos, a HU deverá ser suspensa e o seu uso contraindicado.

- Pessoas com sorologia positiva para hepatite $\mathrm{B} \mathrm{e} \mathrm{C}$ poderão fazer uso de $\mathrm{HU}$ desde que monitorados mensalmente com provas de função hepática.

- Insuficiência renal: embora poucos estudos avaliaram o uso de HU em pacientes com insuficiência renal, recomenda-se o ajuste de dose conforme o valor de depuração da creatinina: $10-50 \mathrm{~mL} / \mathrm{min}$, administrar $50 \%$ da dose; $<10 \mathrm{~mL} / \mathrm{min}$, administrar $20 \%$ da dose. É recomendável a avaliação em conjunto com o nefrologista. Pacientes em 
hemodiálise devem receber HU após o procedimento. É recomendável a avaliação em conjunto com o nefrologista.

- Não há dados para orientação do ajuste de dose em pacientes com insuficiência hepática.

Efeitos adversos relacionados ao uso de $H U$

É importante observar o aparecimento de possíveis efeitos adversos, tais como:

- Neurológico: letargia, cefaleia, tonturas, desorientação e alucinações (raras).

- Gastrintestinal: estomatite, anorexia, náuseas, vômitos, diarreia e constipação.

- Dermatológico: erupção máculo-papular, eritema facial e periférico, alopecia, hiperpigmentação da pele e das unhas, pele seca, ulceração da pele ou agravamento de úlcera já existente.

- Renal: elevação dos níveis séricos de ureia e creatinina. AST).

- Hepático: elevação das aminotransferases (ALT,

- Reprodutivo: oligospermia, azoospermia.

- Mielotoxicidade.

- Efeito teratogênico (confirmado apenas em animais).

- Hiperesplenismo (em crianças).

- Outros: edema, febre, calafrios, mal-estar, astenia.

Qualquer efeito adverso (incluindo alteração laboratorial) deve ser valorizado. No caso de EA de intensidade leve, HU pode ser mantida desde que o paciente seja reavaliado regularmente pelo especialista.

Na vigência de EA moderado ou grave, a administração de HU deve ser suspensa até seu desaparecimento ou normalização laboratorial. A possível reintrodução de $\mathrm{HU}$ dependerá do EA e esta conduta deverá ser tomada conjuntamente entre médico e paciente, e individualizada caso a caso.

Causas de falha do tratamento com $\mathrm{HU}$

- má aderência ao tratamento.

- reserva medular inadequada ou insuficiente.

- fatores genéticos associados que possam interferir na resposta à $\mathrm{HU}$ (farmacogenômica).

\section{Centros de referência}

Conforme já definido na Portaria GM/MS no $822,{ }^{35}$ de 06 de junho de 2001, os serviços de referência em triagem neonatal são os responsáveis pela realização da triagem de hemoglobinopatias de todo recém-nascido no território brasileiro. Todos os demais terão exame de eletroforese de hemoglobina, disponibilizado nas unidades de atenção básica de saúde. O tratamento e acompanhamento dos pacientes com DF deverão ser realizados pelos serviços de referência, que podem ser os hemocentros ou outro centro de atendimento (universitário, hospitalar) que ofereça assistência especializada e integral, e promova prevenção, diagnóstico e tratamento ambulatorial multidisciplinar, assim como tratamento hospitalar, incluindo serviço de emergência, unidade de terapia intensiva e realização de cirurgias eletivas.

\section{Consentimento informado}

É obrigatório que o paciente e/ou seu responsável legal estejam cientes dos potenciais riscos e efeitos colaterais relacionados ao uso da HU preconizado neste Protocolo. Para tanto, o consentimento de participação do protocolo deverá ser formalizado por meio da assinatura de Termo de Consentimento Informado.

\begin{abstract}
Hydroxyurea (HU) is an important major advance in the treatment of sickle cell disease (SCD). Strong evidence supports the effectiveness of $H U$ in adults; severe painful episodes, hospitalizations, number of blood transfusions, and acute chest syndrome are reduced. Although the evidence of its effectiveness in the treatment of children is not as strong, the emerging data is encouraging. Current data on the risks of both short-and longterm HU therapy in adults are acceptable when compared to the risks of untreated SCD. In this article, we present a detailed review of the main aspects of the efficacy, effectiveness, toxicity, and barriers to the use of HU for SCD and propose a clinical protocol and therapeutic guidelines for the use of HU in patients with SCD. Rev. Bras. Hematol. Hemoter.
\end{abstract}

Key words: Sickle cell disease; hydroxyurea; clinical protocol; therapeutic guidelines.

\section{Referências Bibliográficas}

1. Zago MA. Considerações gerais sobre as doenças falciformes. In: Manual de Diagnóstico e Tratamento das Doenças Falciformes (Agência Nacional de Vigilância Sanitária, org.), 2002; pp. 9-11, Brasília: Ministério da Saúde.

2. Cançado RD, Jesus JA. A doença falciforme no Brasil. Rev. Bras. Hematol. Hemoter. 2007;29(3):204-6.

3. Zago MA, Pinto ACS. Fisiopatologia das doenças falciformes: da mutação genética à insuficência de múltiplos órgãos. Rev. Bras. Hematol. Hemoter. 2007;29(3):207-14.

4. Platt OS, Brambilla DJ, Rosse WF, Milner PF, Castro O, Steinberg $\mathrm{MH}$, et al. Mortality in sickle cell disease. Life expectancy and risk factors for early death. N Engl J Med. 1994;330(23):1639-44.

5. Vichinsky E, Hurst D, Earles A, Kleman K, Lubin B. Newborn screening for sickle cell disease: effect on mortality. Pediatrics. 1988;81(6):749-55.

6. Dressler WFC and Sterin R. Uber den Hydroxylharnstoff. Jusutus Liebigs Ann Chem Pharm 1869;150: 242-252. 2000;342(25): 1855-65.

7. Stevens MR. Hydroxyurea: an overview. J Biol Regul Homeost Agents. 1999;13(3):172-5.

8. Charache S, Terrin ML, Moore RD, Dover GJ, Barton FB, Eckert $\mathrm{SV}$, et al. Effect of hydroxyurea on the frequency of painful crises in sickle cell anemia. Investigators of the Multicenter Study of Hydroxyurea in Sickle Cell Anemia. N Engl J Med. 1995; 332 (20):1317-22. 
9. Steinberg MH, Barton F, Castro O, Pegelow CH, Ballas SK, Kutlar A, et al. Effect of hydroxyurea on mortality and morbidity in adult sickle cell anemia: risks and benefits up to 9 years of treatment. JAMA. 2003;289(13):1645-51.

10. Ballas SK, Barton FB, Waclawiw MA, Swerdlow P, Eckman JR, Pegelow $\mathrm{CH}$, et al. Hydroxyurea and sickle cell anemia: effect on quality of life. Health Qual Life Outcomes. 2006;4:59.

11. Krakoff IH, Brown NC, Reichard P. Inhibition of ribonucleoside diphosphate reductase by hydroxyurea. Cancer Res. 1968; 28(8): 1559-65.

12. Figueiredo MS. Agentes indutores da síntese de hemoglobina fetal. Rev bras hematol hemoter, 2007; 29(3):313-315.

13. Covas DT, de Lucena Angulo I, Vianna Bonini Palma P, Zago MA Effects of hydroxyurea on the membrane of erythrocytes and platelets in sickle cell anemia. Haematologica. 2004;89 (3):273-80.

14. Vicari P, Barretto de Mello A, Figueiredo MS. Effects of hydroxyurea in a population of Brazilian patients with sickle cell anemia. Am J Hematol. 2005;78(3):243-4.

15. Canalli AA, Costa FF, Saad ST, Conran N. Granulocytic adhesive interactions and their role in sickle cell vaso-occlusion. Hematology. $2005 ; 10(5): 419-25$

16. Canalli AA, Franco-Penteado CF, Saad ST, Conran N, Costa FF. Increased adhesive properties of neutrophils in sickle cell disease may be reversed by pharmacological nitric oxide donation. Haematologica. 2008;93(4):605-9.

17. Zimmerman SA, Schultz WH, Davis JS, Pickens CV, Mortier NA, Howard TA, et al. Sustained long-term hematologic efficacy of hydroxyurea at maximum tolerated dose in children with sickle cell disease. Blood. 2004;103(6):2039-45.

18. Zimmerman SA, Schultz WH, Burgett S, Mortier NA, Ware RE. Hydroxyurea therapy lowers transcranial Doppler flow velocities in children with sickle cell anemia. Blood. 2007;110(3):1043-7.

19. Ferster A, Tahriri P, Vermylen C, Sturbois G, Corazza F, Fondu P, et $a l$. Five years of experience with hydroxyurea in children and young adults with sickle cell disease. Blood. 2001;97 (11): 3628-32.

20. de Montalembert M, Brousse V, Elie C, Bernaudin F, Shi J, Landais P; French Study Group on Sickle Cell Disease. Long-term hydroxyurea treatment in children with sickle cell disease: tolerance and clinical outcomes. Haematologica. 2006;91(1):125-8.

21. Lanaro C, Franco-Penteado CF, Albuqueque DM, Saad ST, Conran $\mathrm{N}$, Costa FF. Altered levels of cytokines and inflammatory mediators in plasma and leukocytes of sickle cell anemia patients and effects of hydroxyurea therapy. J Leukoc Biol. 2009;85 (2):235-42

22. Hankins JS, Ware RE, Rogers ZR, Wynn LW, Lane PA, Scott JP, et al. Long-term hydroxyurea therapy for infants with sickle cell anemia: the HUSOFT extension study. Blood. 2005; 106 (7):2269-75

23. Hankins JS, Helton KJ, McCarville MB, Li CS, Wang WC, Ware RE. Preservation of spleen and brain function in children with sickle cell anemia treated with hydroxyurea. Pediatr Blood Cancer. 2008;50(2):293-7.

24. Gulbis B, Haberman D, Dufour D, Christophe C, Vermylen C, Kagambega F, et al. Hydroxyurea for sickle cell disease in children and for prevention of cerebrovascular events: the Belgian experience. Blood. 2005;105(7):2685-90.

25.ClinicalTrials.gov Web site. Silent Cerebral Infarct Multi-Center Clinical Trial. http: //www.clinicaltrials.gov/ct/show/ NCT00072761. Accessed January 20, 2009. ClinicalTrials.gov Web site. Stroke With Transfusions Changing to Hydroxyurea

26. Mueller BU. When should hydroxyurea be used for children with sickle cell disease? Pediatrics. 2008;122(6):1365-6.
27. Kinney TR, Helms RW, O'Branski EE, Ohene-Frempong K, Wang $\mathrm{W}$, Daeschner C, et al. Safety of hydroxyurea in children with sickle cell anemia: results of the HUG-KIDS study, a phase I/II trial. Pediatric Hydroxyurea Group. Blood. 1999;94(5):1550-4.

28. (SWiTCH). http://www.clinicaltrials.gov/ct/show/NCT00122980. Accessed January 20, 2009.

29. Brawley OW, Cornelius LJ, Edwards LR, Gamble VN, Green BL, Inturrisi $\mathrm{C}$, et al. National Institutes of Health Consensus Development Conference statement: hydroxyurea treatment for sickle cell disease. Ann Intern Med. 2008;148(12):932-8. http:// consensus.nih.gov/aboutcdp.htm.

30. Lanzkron S, Strouse JJ, Wilson R, Beach MC, Haywood C, Park H, et al. Systematic review: Hydroxyurea for the treatment of adults with sickle cell disease. Ann Intern Med. 2008;148(12):939-55.

31. Strouse JJ, Lanzkron S, Beach MC, Haywood C, Park H, Witkop $\mathrm{C}$, et al. Hydroxyurea for sickle cell disease: a systematic review for efficacy and toxicity in children. Pediatrics. 2008;122(6): $1332-42$.

32. Portaria no 872 do Ministério da Saúde, de 6 de novembro de 2002. Protocolo Clínico e Diretrizes Terapêuticas - Doença Falciforme - Hidroxiureia.

33. Bomediano VH, Pinheiro VRP, Anjos AC, et al. Protocolo prospectivo para uso de hidroxiureia em crianças com doença falciforme. Centro Integrado de Pesquisas Oncológicas na Infância (CIPOI), Unicamp, 1995.

34. Chaine B, Neonato MG, Girot R, Aractingi S. Cutaneous adverse reactions to hydroxyurea in patients with sickle cell disease. Arch Dermatol. 2001;137(4):467-70.

35. Portaria GM/MS n ${ }^{\circ} 822$, de 06 de junho de 2001. Art. $1^{\circ}$ Instituir, no âmbito do Sistema Único de Saúde, o Programa Nacional de Triagem Neonatal / PNTN.

Avaliação: Editor e dois revisores externos

Conflito de interesse: sem conflito de interesse

Recebido: 24/03/2009

Aceito após modificações: 01/07/2009 